\title{
ICA \& Wavelet as a Method for Speech Signal Denoising
}

\author{
Ms. Niti Gupta ${ }^{1}$ and Dr. Poonam Bansal ${ }^{2}$
}

\begin{abstract}
Speech signal denoising is a highly researched topic and here in this paper we discuss denoising using Independent Component analysis and Wavelet transform, Wavelet Thresholding is applied to noise dominated signal and both method of thresholding soft and hard is used. Independent Component analysis is a separation method which is dependent on blind source method. In this paper ICA is discussed and applied for speech denoising. Simulation results indicate that both the proposed method exhibit superior denoising performance but performance of ICA was better compared to Wavelet

Index Terms: Speech signal denoising, wavelet, independent component analysis, SNR, Hard Thresholding, Soft Thresholding.
\end{abstract}

\section{INTRODUCTION}

Noise is most clinical and discussed problem among signal processing. Noisy signal can be expressed as (1) where $s(t)$ is the speech signal free of noise, $n(t)$ is noise whose distribution is obeyed by $\mathrm{N}(0,1), \sigma$ is the variance. The aim or the goal is to get a denoised signal estimation.

$X(t) \square s(t) \square \sigma n(t)$

The estimation is based on the statistical property of original signal. To solve these problems linear filters are generally used as they are easy to implement. But these are not effective when $\sigma$ is not known [1]. So based on this other approaches has been proposed. Method based on Wavelet thresholding, ICA, Empirical mode decomposition .EMD [2] was proposed by Huang were signal could be decomposed into several functions based on an iterative method called shifting. In wavelet, signal is transformed to wavelet domain first .Speech signal is always focused at fewer component and at high amplitude with noise being spread across low amplitude [3]. Based on the threshold wavelet coefficient are compared and are modified. With inverse transform denoised signal is obtained. EMD or wavelet has a shortcoming if the Signal to Noise ratio is low [4]. Research has been going how to how to combine both EMD and wavelet [5]. ICA is one of the famous blind source separation method, which is dedicated to recover unknown signals which are mutually

\footnotetext{
${ }^{1}$ Computer science departmentJagannath University Jaipur, India

${ }^{2}$ Computer science and engineering Maharaja Surajmal institute of Technology Janakpuri, Delhi, India
} 
independent without the knowledge of coefficient [6]. Hence if signal can be denoised through ICA a huge improvement in SNR can be achieved [7]. Some researcher have given a approach on the basis of EMD and ICA [9]. We have proposed a generic idea of speech signal denoising which uses wavelet and ICA. In this paper, we have modified a part of the ICA algorithm. And we have used both Hard and soft thresholding to check the performance. The detail analysis of the results is demonstrated with the result, where denoising is exhibited superior performance in half a half shorter running time

\section{BASIC THEORY}

\section{A. Wavelet Thresholing}

DWT or the Discrete Wavelet Transform breaks the signal into number of components using mother wavelet[4]. The signal is expressed in terms of linear sum of wavelet and wavelet coefficients, So choosing proper mother wavelet is very important [3]. Wavelet thresholding can be described as follows (2-8). Where $\mathrm{x}$ is decomposed into wavelet domain using transformation matrix, where transform matrix is $\boldsymbol{U}$

$$
\begin{gathered}
\mathbf{x} \square\left[x_{1}, x_{2}, \ldots, x_{N}\right] \\
\mathrm{c}=\mathrm{Ux}
\end{gathered}
$$

where Wavelet coeffiecnts are given by $\mathbf{c} \square\left[c_{1}, c_{2}, \ldots c_{N}\right]$.

Two types of thresholding are present which can be considered as a rule of thresholding referred as hard and soft thresholding
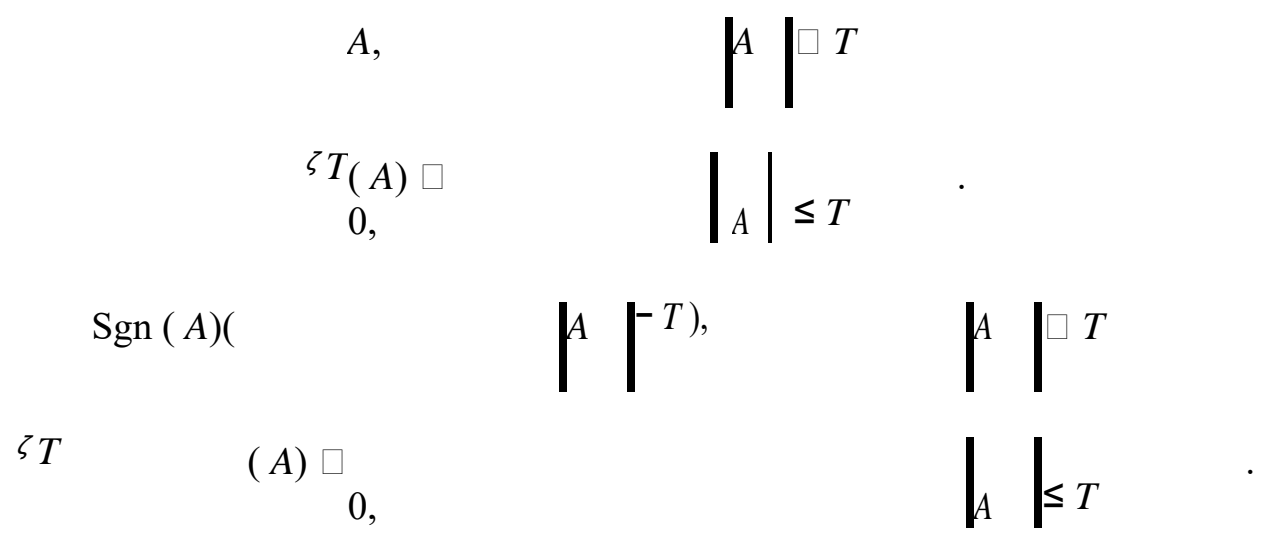

Here $A$ is referred to as amplitude of wavelet coefficient, and $\zeta_{T} \square A \square$ as after thresholding amplitude and $T$ is defined as the thresholding value. With Soft thresholding discontinuity in the denoised signal does not exist, so for application such as speech processing soft thresholding is preferred. After using either soft or hard thresholding denoised signal can be estimated using Inverse transform.

$$
\mathrm{X}^{\wedge}=\mathrm{U}^{\mathrm{T}} \mathrm{c}
$$


Where $U^{T}$ is the transposition matrix of $U$ and $x$. The performance of denoising is based on the thresholding method selected and wavelet has lower response if the SNR is very low.

\section{B Independent Component analysis (ICA method)}

It is a statistical method used for transforming a vector which is statistically independent [16] . P. Comon introduced a process of optimization or a learning rule[17]. Hyvianne introduced a object function. Considering linear mixing, the signal can be represented as

$$
\mathrm{M}=\mathrm{AS}
$$

Where $\mathbf{M}=\left[m_{1}, m_{2}, m_{p}\right]$ is a Summation of received signals $\mathrm{S}=\left[s_{1}, s_{2}, s_{\mathrm{n}}\right]$ with $\mathrm{S}$ as the source signal and mixing matrix represented by A. Inverse of A if estimated accurately approximate to be $\mathbf{W}=\mathbf{A}^{\mathbf{- 1}}$, then the source signals could be recovered by

$$
\mathrm{S}=\mathrm{W} \cdot \mathrm{M}=\mathrm{W} \cdot(\mathrm{AS})==\mathrm{S}
$$

It is noticeable that Common ICA is capable of recovering all components which are independent because of unmixing matrix $\mathrm{W}$ which is present in the learning rule [11]. This makes it inefficient when only few signal source are required or desired. But the ICA can be modified to extract single component whose frame work is represented in

Maximize $K \square y \square \approx \rho E \square G \square y \square \square-E \square G \square v \square \square{ }^{2}{ }_{(16)}$ subject to $g \square \mathbf{w} \square \leq 0, h \square \mathbf{w} \square \square E \square$ $y^{2} \square-1 \square 0$

where $K \square y \square$ is a reliable approximation. The inequality $\mathrm{g} \square \mathbf{w} \square$ can be exploited to incorporate prior information of the desired signal into the ICA learning rule. The equality constraint $h \square \mathbf{w} \square$ is used to bound $K \square y \square$ and the weight vector $\mathbf{w}$.

\section{SIMUALATION RESULTS}

To verify the validity of simulations on the auto-correlation criterion, the output SNR, the correlation coefficient and the running time are performed. Then the results are analysed and compared to the methods aforementioned.

\section{A. The Auto-correlation Criterion}




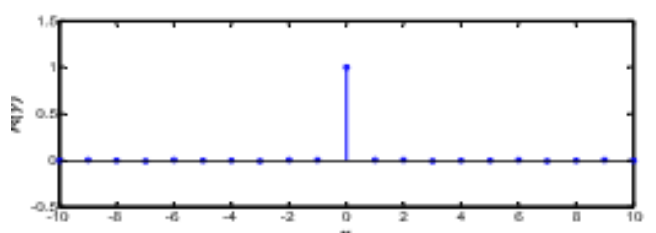

(a)

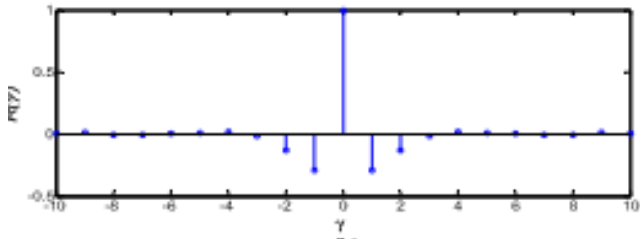

(b)

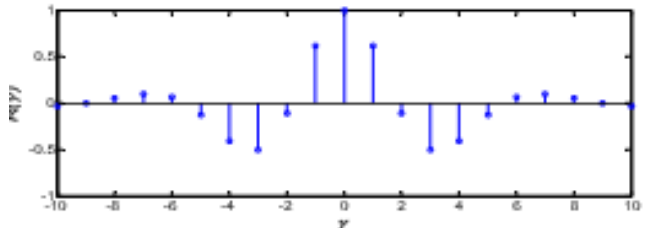

(c)

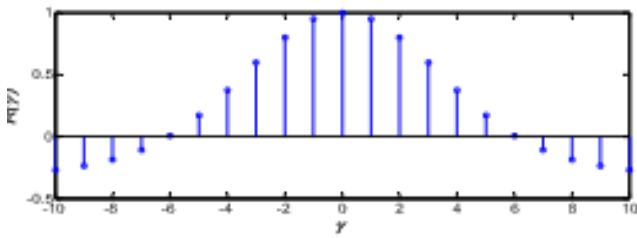

(d)

Figure 2. (a)The auto-correlation of Gaussian white noise (b)The auto-correlation of $\operatorname{imf}_{1}$ (c)The auto-correlation of $\operatorname{imf}_{2}$ (d)The auto-correlation of $i m f_{3}$

The auto-correlation of the Gaussian white noise and the frontal three IMFs from the decomposition of noisy speech when $\mathrm{SNR}=-3 \mathrm{~dB}$ are shown in Fig. 2. In Fig. 2 (b) and (c), the autocorrelation properties of the frontal two IMFs indicate that they are noise-dominated. In Fig. 2 (d), the short-time auto-correlation properties of speech signal is dominated, which indicates $\mathrm{imf}_{3}$ mainly contains speech signal.

\section{B. Waveform}

The waveforms of the original speech signal, the noisy signal corresponding to $\mathrm{SNR}=-3$ signals by different approaches demonstrated in Fig. 4.(d)and(e) demonstrate that using mother wavelet will lead to different denoising performance. It's obvious that db6 mother wavelet outperforms sym8 as for this chosen speech signal. In EMD-wavelet-ICA approach,the selection is also unavoidable inStep2.EMD-wavelet-ICA utilizes EMD other than wavelet decomposition to 
analysis the signal in Step1, hence the of selecting appropriate mother brought to minimum. For simplicity, we mother wavelet in the following simulations.(c),(d),(e),(f) and(g)show that by simply EMD or wavelet considerable residual noise, while EMD-ICA and EMD-wavelet perform much better, but not extraordinary satisfactory. As can be seen from Fig. 3, our method exhibits a superior performance in denoising.

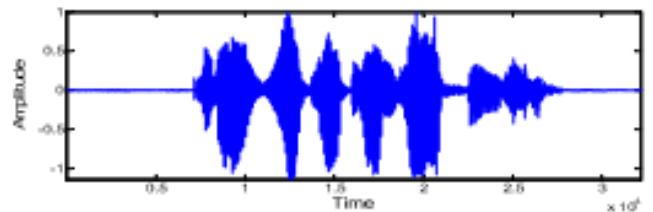

(a)

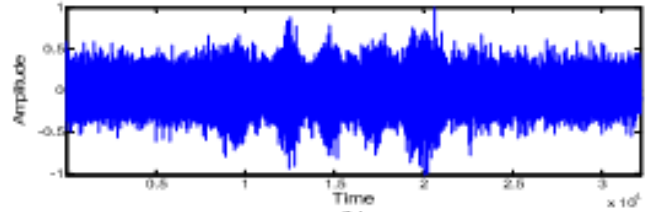

(b)

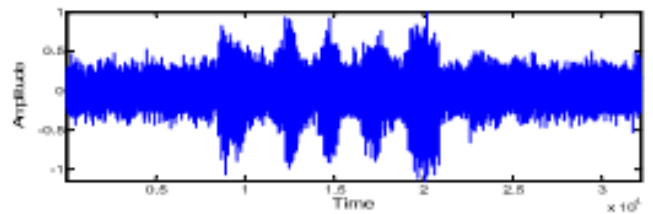

(c)

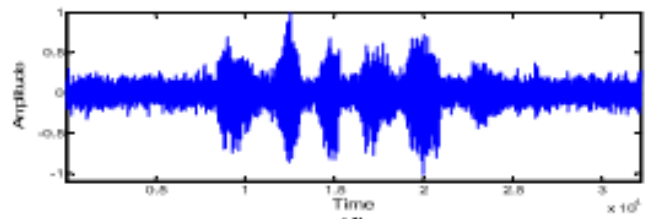

(d)

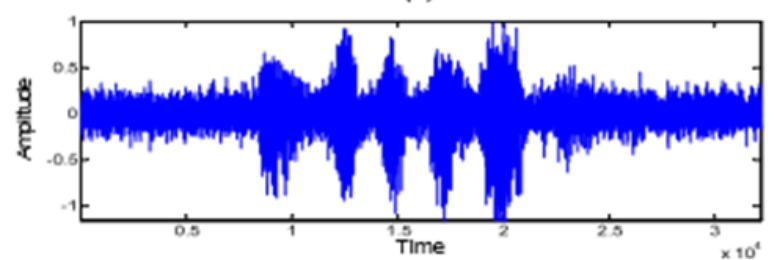

(e)

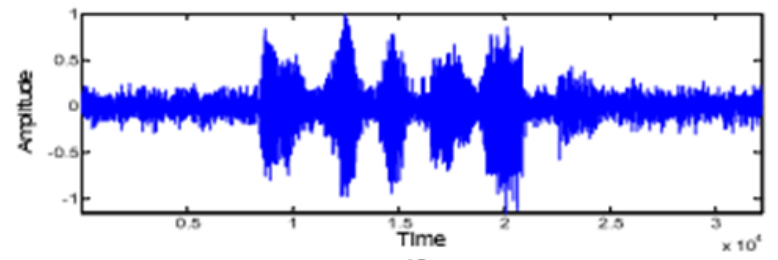

(f)

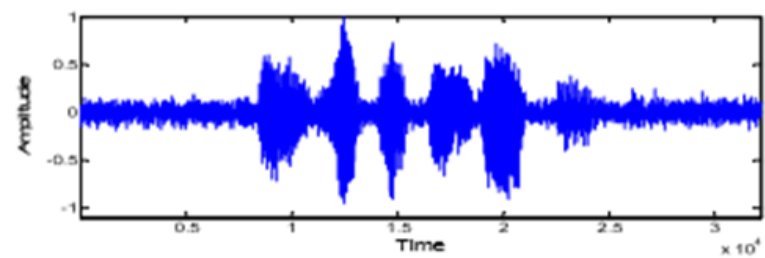

(g)

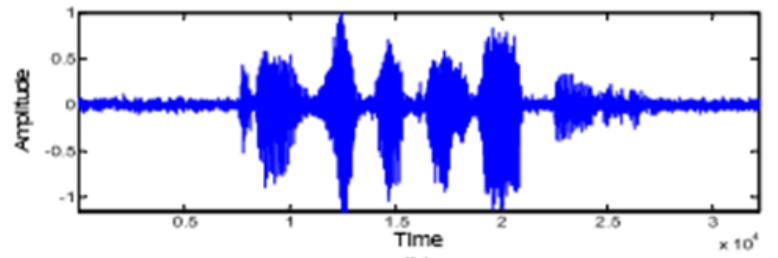

(h)

Figure 3. (a)The waveform of the clean speech signal (b)The waveform of the noisy signal corresponding to $\mathrm{SNR}=-3 \mathrm{~dB}$ (c)The waveform of the denoised result by EMD (d)The waveform of the denoised result by the wavelet (db6 mother wavelet) e)The waveform of the denoised result by the wavelet (sym8 mother wavelet) (f)The waveform of the denoised result by the EMD-ICA (g)The waveform of the denoised result by the EMD-wavelet (h)The waveform of the denoised result by the

\section{Correlation Coefficient}

EMD-wavelet-ICA

The value of correlation indicates how much the denoised signal resembles its original version.

$$
\rho \square s^{\wedge}, s \square \square \quad \frac{\operatorname{cov}\left(s^{\wedge}, s\right)}{\sqrt{\operatorname{cov}} \quad s^{\wedge}, s^{\wedge} \cdot \operatorname{cov} s, s}
$$


This criterion cancels out the influence of amplitude in ICA, the closer the correlation coefficient to 1 , the better the denosing performance is. For each SNR value, 100 independent noise sequences are generated and the correlation coefficients are calculated in Fig. 5.From Fig. 5, performance of the proposed method remains good when signal-to-noise is low, while other methods degrade substantially. When input $\mathrm{SNR}=-5 \mathrm{~dB}$, the correlation coefficient of the noisy speech and the speech signal is 0.4903 , and that of the denoised result by simply EMD approaches and the speech signal becomes 0.5813 . In an ascending order, the correlation coefficient reaches up to $0.6510,0.6539$, 0.6686 and 0.7237 respectively by wavelet, EMD-wavelet, EMD-ICA and EMD-wavelet-ICA approaches. The proposed method can achieve almost $47 \%$ performance enhancement compared to the noisy speech.

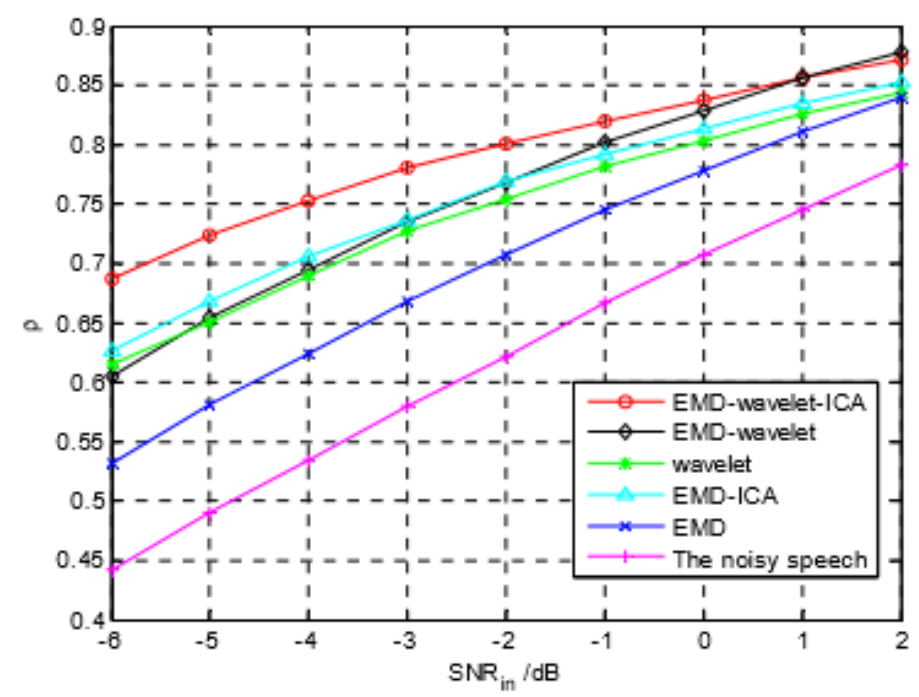

Figure 5. The relationship between correlation coefficient and input SNR

\section{CONCLUSION}

Denoising Method- based on wavelet analysis for removal of noise from sound signal may lead to loss of the data and there may be still remnant artifact in the collected samples. It is known that signals with higher PSNR and SNR and low MSE are less noisy signals. By looking at the various evaluation parameters like MSE, PSNR, SNR calculated, it is concluded that wavelet method gave the best denoising result with its multi resolution capacities. Wavelet transform analyses the signals in both time and frequency domain and also signals with low noise amplitudes can be removed from the signals by selecting the best wavelet to decompose the signal. In wavelet transform we decompose only the low pass components of the signals. ICA method was based on the blind source separation and it could analyze the signals only in time domain the frequency components of the signal therefore could not be analyzed. For ICA - two random signals are 
generated and they are mixed with noise. ICA algorithm shows that it is efficient method to separate multiple signals from noise as well as from each other.

\section{REFERENCES}

[1] K. Khaldi, A. O. Boudraa, A. Alouane, A. Bouchikhi. M. T-H. Alouane, and E-H. S. Diop, "Speech signal noise reduction by EMD," International Symposium on Communications Control and Signal Processing, Mar. 2008, pp.1155-1158.

[2] N. E. Huang et al. "The empirical mode decomposition and the Hilbert spectrum for nonlinear and non-stationary time series analysis," Proc. R. Soc. Lond. A, vol. 454A, pp. 903-995, Mar.1998.

[3] A. O. Boudraa, J .C .Cexus, and Z. Saidi, "EMD-based signal noise reduction," International Journal of Signal Processing, vol. 1, Jan. 2004, pp.33-37.

[4] M. A. Kabir and C. Shahnaz, "Denosing of ECG signals based on noise reduction algorithms in EMD and wavelet domains," Biomedical Signal Processing and Control, May. 2012, pp.481-489.

[5] Y. Kopsinis and S. McLaughlin. "Development of EMD-based denoising methods inspired by wavelet thresholding," IEEE Transactions on Signal Processing, vol. 57, Apr. 2009, pp. 13511362.

[6] A. Hyvärinen and E. Oja, "Independent component analysis: algorithms and applications," Neural Networks, vol. 13, 2000, pp. 411-430.

[7] H. Li and Y. Sun, "Denoising by ICA based on EMD virtual Channel," Journal of Beijing University of Posts and Telecommunications, vol.30, Oct.2007, pp.33-36.

[8] M. E. Davies and C. J. James, "Source separation using single channel ICA," Signal Processing, vol. 87, Aug. 2007, pp. 1819-1832.

[9] B. Mijović, M. D. Vos, I. Gligorijević, J. Taelman, and S. V. Huffel, "Source separation from single-channel recordings by combining empirical-mode decomposition and independent component analysis," IEEE Transactions on Biomedical Engineering, vol. 57, Sept. 2010, pp. 2188-2196.

[10] J. Liu, S. Yang, and S. Piao, "The single channel seismic-acoustic signal blind-source separation method based on EEMD," Journal of Harbin Engineering University, vol.32, Feb.2011, pp. 194-199.

[11] W. Lu and J. C. Rajapakse, "ICA with reference," Proc. 3rd International Conference on Independent Component Analysis and Blind Source Separation, 2001, pp.120-125.

[12] Y. Qi, G. Wang, and M. Yu, "A noise reduction method for speech signal combining ICA-R and EMD-wavelet," International Conference on Mechatronic Sciences, Electric Engineering and Computer, Dec. 2013. 